\title{
Systematic review of the evidence on the cost-effectiveness of pharmacogenomics-guided treatment for cardiovascular diseases
}

\author{
Ye Zhu, MD, PhD ${ }^{1,2}$, Kristi M. Swanson, MS ${ }^{1}$, Ricardo L. Rojas, BA ${ }^{3}$, Zhen Wang, PhD ${ }^{1,4}$, \\ Jennifer L. St. Sauver, $\mathrm{PhD}^{1,5}$, Sue L. Visscher, $\mathrm{PhD}^{1}$, Larry J. Prokop, $\mathrm{MLS}^{6}$, \\ Suzette J. Bielinski, MEd, $\mathrm{PhD}^{5}$, Liewei Wang, $\mathrm{MD} \mathrm{PhD}^{7}$, Richard Weinshilboum, $\mathrm{MD}^{7}$ and \\ Bijan J. Borah, PhD (1) ${ }^{1,2}$
}

Purpose: To examine the evidence on the cost-effectiveness of implementing pharmacogenomics (PGx) in cardiovascular disease (CVD) care.

Methods: We conducted a systematic review using multiple databases from inception to 2018. The titles and abstracts of costeffectiveness studies on PGx-guided treatment in CVD care were screened, and full texts were extracted.

Results: We screened 909 studies and included 46 to synthesize. Acute coronary syndrome and atrial fibrillation were the predominantly studied conditions (59\%). Most studies (78\%) examined warfarin-CYP2C9/VKORC1 or clopidogrel-CYP2C19. A payer's perspective was commonly used (39\%) for cost calculations, and most studies (46\%) were US-based. The majority (67\%) of the studies found PGx testing to be cost-effective in CVD care, but costeffectiveness varied across drugs and conditions. Two studies examined PGx panel testing, of which one examined pre-emptive testing strategies.

Conclusion: We found mixed evidence on the cost-effectiveness of PGx in CVD care. Supportive evidence exists for clopidogrelCYP2C19 and warfarin-CYP2C9/VKORC1, but evidence is limited in other drug-gene combinations. Gaps persist, including unclear explanation of perspective and cost inputs, underreporting of study design elements critical to economic evaluations, and limited examination of PGx panel and pre-emptive testing for their costeffectiveness. This review identifies the need for further research on economic evaluations of PGx implementation.

Genetics in Medicine (2020) 22:475-486; https://doi.org/10.1038/s41436019-0667-y

Keywords: economic evaluation; cost-effectiveness; pharmacogenomics; cardiovascular disease; disease management

\section{INTRODUCTION}

Pharmacogenomics (PGx) examines how variations in an individual's DNA sequence affect drug metabolism and response, including occurrence of adverse drug events and impact on treatment effectiveness. ${ }^{1,2}$ It differs from traditional diagnostic genetic/genomic testing, which looks at the associations between changes in a person's DNA sequence and diseases. ${ }^{3}$ Thus, PGx may facilitate a personalized approach to disease management by identifying the safest and most effective treatment options for an individual. Cardiovascular disease (CVD) treatment is at the forefront of PGx-guided therapy. Being the leading cause of death worldwide, CVD contributes significantly to the increasing health economic burden. In 2016,
CVD accounted for $31 \%$ of all deaths globally (17.9 million people). ${ }^{4}$ Approximately $\$ 555$ billion in CVD-related direct and indirect health-care costs were incurred in the United States in 2016. These costs are projected to increase to $\$ 1.1$ trillion by the year 2035. ${ }^{5}$ A number of drug classes exist to reduce CVD risk, but there is significant variation in treatment response. ${ }^{6}$ In addition to the variation that can be attributed to various sociodemographic characteristics, genetic determinants of drug response have been identified, which can affect the way drugs are metabolized, absorbed, and distributed. ${ }^{1,6-8}$ Therefore, genetic information can be used to inform risk factor identification, drug dose responses, side effects management, and outcome prediction. ${ }^{9-11}$

\footnotetext{
${ }^{1}$ Robert D. and Patricia E. Kern Center for the Science of Health Care Delivery, Mayo Clinic, Rochester, MN, USA; ${ }^{2}$ Division of Health Care Policy and Research, Department of Health Sciences Research, Mayo Clinic, Rochester, MN, USA; ${ }^{3}$ Division of Biomedical Statistics and Informatics, Department of Health Sciences Research, Mayo Clinic, Rochester, MN, USA; ${ }^{4}$ Evidence-Based Practice Center, Mayo Clinic, Rochester, MN, USA; ${ }^{5}$ Division of Epidemiology, Department of Health Sciences Research, Mayo Clinic, Rochester, MN, USA; ${ }^{6}$ Library Public Services, Mayo Clinic, Rochester, MN, USA; ${ }^{7}$ Division of Clinical Pharmacology, Department of Molecular Pharmacology and Experimental Therapeutics, Mayo Clinic, Rochester, MN, USA. Correspondence: Bijan J. Borah (Borah.Bijan@mayo.edu)

These authors contributed equally: Ye Zhu, Kristi M. Swanson
} 
PGx-guided drug prescribing holds enormous potential for individualizing CVD management and reducing the risks of drug-related adverse events. Research has shown that it is often a combination of gene variants that accounts for variations in disease progression and treatment response. The Right Drug, Right Dose, Right Time-Using Genomic Data to Individualize Treatment (RIGHT) study examined five PGx genes (SLCO1B1, CYP2C19, CYP2C9, VKORC1, and CYP2D6), four of which had CVD implications; the study showed that approximately $58 \%$ of subjects carried clinically actionable genetic variants (i.e., presence of gene variants that warrant a dose or drug change) for three or more of the five genes studied. ${ }^{12}$ Furthermore, an additional $31 \%$ of patients had two actionable genetic variants, and $10 \%$ of patients had only one. A safety net health-care system in Indiana further demonstrated the impact that PGx implementation would have by examining prescriptions for 30 targeted medications with known PGx interactions. Approximately $20 \%$ of these patients were being treated with two or more of the targeted medications. Three CVD medications (clopidogrel, warfarin, and simvastatin) were included in this study and accounted for $22 \%$ of the prescriptions identified. ${ }^{13}$ Recognizing the importance of PGx-guided treatment, the US Food and Drug Administration (FDA) has recently provided guidance on the inclusion of appropriate PGx information in drug labels of over 362 drugs. ${ }^{14-16}$

Despite the literature supporting the usefulness of PGxguided therapy, evidence of its effectiveness in CVD treatments is equivocal. While some studies show PGxguided treatment to be effective compared with standard-ofcare treatments, ${ }^{17,18}$ others did not find it to be effective in clinical practice. ${ }^{19-22}$ For example, a randomized trial by the European Pharmacogenetics of Anticoagulant Therapy (EUPACT) found a significant association between PGx-guided warfarin dosing and the reduction in the time it took patients to reach the therapeutic range for their international normalized ratio (INR). ${ }^{23}$ Stergiopoulos and Brown, however, conducted a meta-analysis that showed PGx-guided warfarin dosing strategies to be ineffective at increasing the percentage of time in the therapeutic range for INR. ${ }^{19}$ Such heterogeneity in empirical evidence has caused controversy around this topic regarding the use of different study strategies (e.g., time to therapeutic range, or percentage of time in the therapeutic range), target populations (e.g., races, gender, age), and drug selection (warfarin versus its analogues), as well as other practice-related factors (practice variations), including the type and timing of PGx testing (e.g., pre-emptive testing versus reactive genotyping). ${ }^{15,24,25}$

Considering the clinical and economic burden of CVD, there is a multitude of empirical evidence examining the implications of PGx-guided treatment in CVD care. ${ }^{26-28}$ This growth in clinical implementation, coupled with potential heterogeneity of empirical evidence, necessitates efforts to clearly summarize the findings and recommendations regarding PGx-guided treatment for CVD. Synthesizing and evaluating the evidence regarding the cost-effectiveness of
PGx is particularly important given the enormous potential PGx holds in reducing adverse drug reactions, improving patient outcomes, and reducing potentially avoidable costs in disease management. In this study, we performed a comprehensive systematic literature review of economic evaluations examining the implementation of PGx in CVD care. The objective of this study is to review and update the current knowledge and gaps with regard to the costeffectiveness of PGx in CVD, and provide information for the considerations of implementing PGx-guided therapy into clinical practice.

\section{MATERIALS AND METHODS}

\section{Search strategy}

We conducted a comprehensive literature search in several databases from each database's inception to 8 August 2018. The databases included Ovid MEDLINE and Epub Ahead of Print, In-Process \& Other Non-Indexed Citations, and Daily; Embase; Cochrane Central Register of Controlled Trials; Cochrane Database of Systematic Reviews; NHS Economic Evaluation Database 1st Quarter 2016; and Scopus. An experienced librarian (L.J.P.) designed and carried out the search strategy with input from the study's principal investigators (Y.Z., K.M.S., and B.J.B.). The search strategy is included in Appendix 1.

\section{Study selection}

We developed a predetermined list of exclusions (Fig. 1) including (1) non-English study, (2) full-text not available, (3) article type (e.g., conference paper/abstract, commentary, book chapter, etc.), (4) genetic testing was not used to identify actionable gene-drug relationships or to guide choice of pharmacologic treatment, (5) no economic evaluation was presented in the study results, (6) CVD was not the main focus of the study (this exclusion criterion also encompasses studies that reported cardiovascular complications as secondary to treatment of other disease systems), and (7) the study intervention was not applied to human subjects. Additionally, duplicated studies were excluded from the final selection.

Selection of articles involved a two-step screening process. In the first step, two independent reviewers (Y.Z. and K.M.S.) screened the title and abstract of each article identified through the search strategy for relevancy to the area of PGx and CVD, with disagreements resolved by a third independent reviewer (B.J.B.). In the second step, the research team retrieved the full texts for the studies remaining, and two independent reviewers (Y.Z. and K.M.S.) assessed the studies for inclusion using the preidentified exclusion criteria. The lead reviewers (Y.Z. and K.M.S.), along with a third independent reviewer (B.J.B.), resolved disagreements in the selection of articles after full-text screening through discussion and consensus.

\section{Data extraction}

Data extraction focused on four domains of information: (1) study design (type of economic evaluation, study perspective, time horizon, etc.), (2) study population (target population, disease or condition, and setting), (3) target treatments 


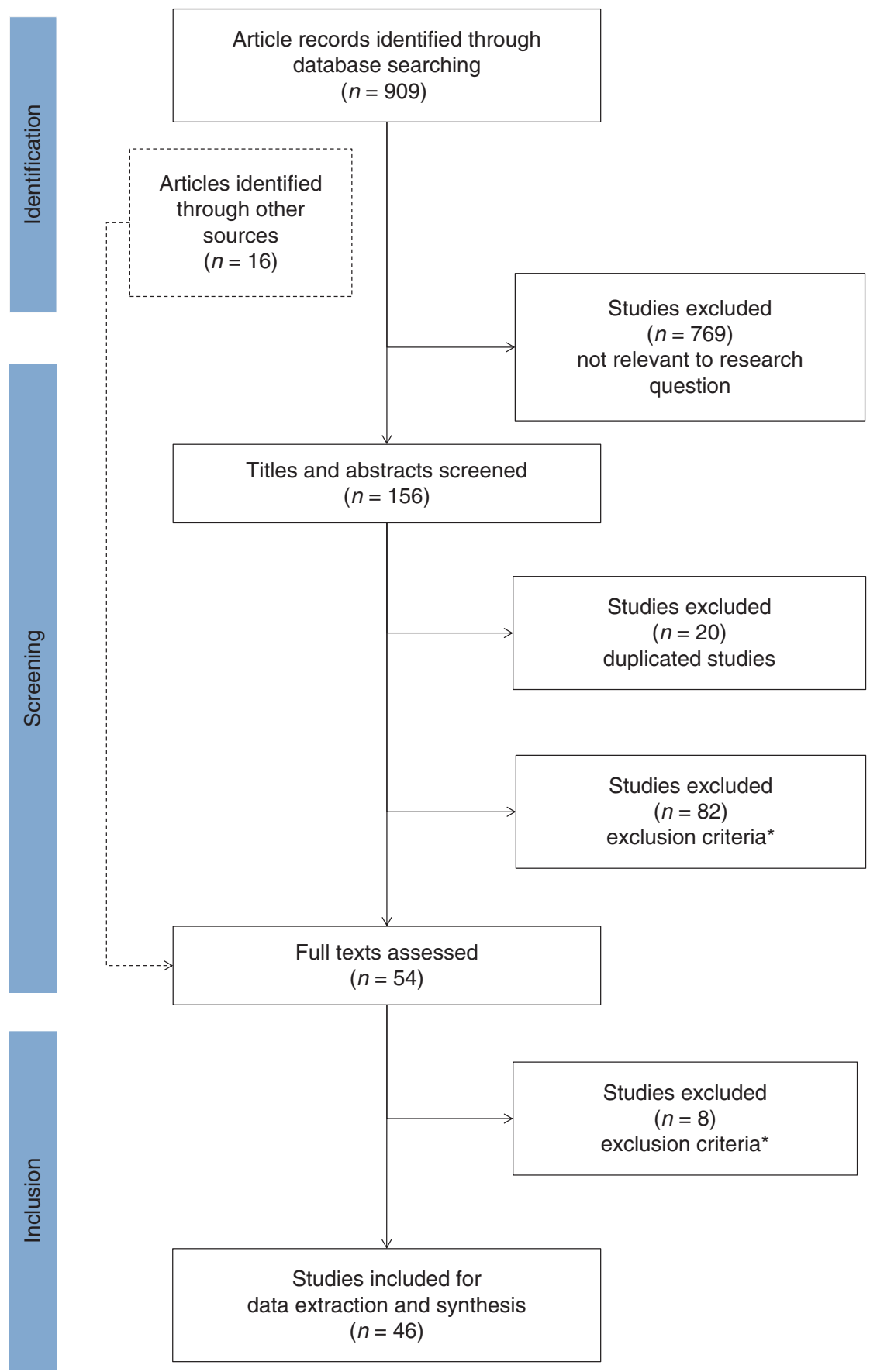

Fig. 1 Flowchart of the literature screening. *Exclusion criteria: 1) non-english study. 2) full text not available. 3) article types: conference paper/abstract, comment, review, short survey, editorial, note, letter, legal case, congress, newspaper articles, book chapter, guideline, erratum, study protocols. 4) not relevant to pharmacogenomics, e.g. genetic diagnostic testing, 5) no economic evaluation, 6) not cardiovascular diseases, 7) intervention not applied to human subjects.

(genetic biomarker, drug therapies, and treatment arms), and (4) outcomes (costs and effectiveness of the drugs/treatments, incremental cost-effectiveness ratios, and recommendations). Study perspective refers to the perspective of the potential decision maker that will eventually use the study results, and therefore costs, effectiveness, and other parameter information included in the study will need to be measured from that specific perspective. For example, societal perspective will require consideration of all parties that will be impacted by the intervention in consideration, and therefore all outcomes and costs, including both direct and indirect costs, will be assessed from that perspective. ${ }^{29}$ Similarly, a health sector perspective (e.g., payer) will incorporate a narrower view and include only outcomes and costs pertinent to that specific health sector. ${ }^{29}$ We also collected additional information related to the publication (authors, title, year, and funding 
declaration) to assess study quality. Two reviewers were assigned to extract data independently from each of the articles that passed the selection criteria (Y.Z., K.M.S., R.L.R., and B.J.B.), and consensus was used to resolve any disagreement.

\section{Quality of reporting assessment}

We assessed the quality of the studies included in the review using Quality of Health Economic Studies (QHES) scores, a weighted grading system that measures the quality of studies based on whether the study reports important design and results elements. ${ }^{30}$ We evaluated each study against 16 domains, including reporting of study characteristics (e.g., funding resources, patient subgroups), study design (e.g., variable estimation, perspective), as well as results and conclusions (e.g., bias assessment, limitations, and recommendations). The QHES score can vary from 0 to 100 , with 100 indicating a perfect score.

\section{Presentation of results}

Due to the heterogeneity present in the study designs across the sample, we synthesized the results using a narrative method. Results include a description of the study selection process, summary statistics illustrating the characteristics of the included studies (e.g., year, country/region, condition studied, etc.), an overview of the various study strategies employed, and a summary of the cost-effectiveness results and final conclusions regarding implementation of PGx testing.

\section{Study selection}

\section{RESULTS}

The search strategy identified a total of 909 studies, and we identified an additional 16 studies in the title and abstract screening process (Fig. 1). We excluded 769 studies during the first step of screening, resulting in 156 studies included for full-text review. After applying exclusion criteria in the second step of the selection process, we identified 46 studies for data extraction.

\section{Summary of the study characteristics}

Over half $(n=26,56 \%)$ of the studies were published between 2014 and 2018 , with only a handful $(n=5,11 \%)$ of studies appearing prior to 2009. US patients were the most commonly targeted population $(n=21,46 \%)$, but there was global representation in our sample, including studies from the Netherlands, Canada, the United Kingdom, and China (Table 1). The most common CVD conditions studied were atrial fibrillation (AF) and acute coronary syndrome (ACS), accounting for $30 \%(n=14)$ and $28 \%(n=13)$ of the studies, respectively. The remaining studies covered a variety of general CVDs (e.g., arterial sclerosis, hypertension, chronic heart disease), more narrow CVD subgroups (e.g., ST-elevated myocardial infarction), or multiple CVD combinations (e.g., stroke and acute myocardial infarction). A small subset of studies $(n=2,4.3 \%)$ focused on procedures, limiting analysis
Table 1 Summary of study characteristics and strategies $(N=46)$

Study characteristics $n(\%)$

\begin{tabular}{|c|c|}
\hline \multicolumn{2}{|l|}{ Year } \\
\hline 2004-2008 & $5(11 \%)$ \\
\hline 2009-2013 & $15(33 \%)$ \\
\hline 2014-2018 & $26(56 \%)$ \\
\hline \multicolumn{2}{|l|}{ Country } \\
\hline United States & $21(46 \%)$ \\
\hline Netherlands & $6(13 \%)$ \\
\hline Canada & $4(9 \%)$ \\
\hline Australia & $2(4 \%)$ \\
\hline Other countries/regions ${ }^{b}$ & $10(22 \%)$ \\
\hline No statement & $3(7 \%)$ \\
\hline \multicolumn{2}{|l|}{ Disease diagnosis or indication } \\
\hline Acute coronary syndrome (ACS) & $13(28 \%)$ \\
\hline Atrial fibrillation (AF) & $14(30 \%)$ \\
\hline Other diseases/patient subgroups ${ }^{c}$ & $13(28 \%)$ \\
\hline No statement & $6(13 \%)$ \\
\hline \multicolumn{2}{|l|}{ Drug category (associated genes) } \\
\hline P2Y12 inhibitors (CYP2C19) & $16(35 \%)$ \\
\hline Antithrombotic (CYP2C9, VKORC1) & $20(43 \%)$ \\
\hline ACEIS (MME, XPNPEP2) & $2(4 \%)$ \\
\hline Statin (and ezetimibe) (KIF6, Taq1B) & $4(9 \%)$ \\
\hline Drug panels & $2(4 \%)$ \\
\hline Others $^{\text {d }}$ & $2(4 \%)$ \\
\hline \multicolumn{2}{|l|}{ Testing timing } \\
\hline After treatment plan made & $42(91 \%)$ \\
\hline After treatment started & $2(4 \%)$ \\
\hline Pre-emptive & $1(2 \%)$ \\
\hline No statement & $1(2 \%)$ \\
\hline \multicolumn{2}{|l|}{ Funding support } \\
\hline Public & $17(37 \%)$ \\
\hline Nonprofit organization (NPO) & $2(4 \%)$ \\
\hline Private & $5(11 \%)$ \\
\hline Combinations $^{\mathrm{e}}$ & $7(15 \%)$ \\
\hline None & $7(15 \%)$ \\
\hline No statement & $8(17 \%)$ \\
\hline Study strategies & $n(\%)$ \\
\hline \multicolumn{2}{|l|}{ Cohort type } \\
\hline Hypothetical & $41(89 \%)$ \\
\hline Observational & $1(2 \%)$ \\
\hline Randomized controlled trial & $4(9 \%)$ \\
\hline \multicolumn{2}{|l|}{ Perspective } \\
\hline Payer $^{f}$ & $18(39 \%)$ \\
\hline Health-care provider & $8(17 \%)$ \\
\hline Societal & $4(9 \%)$ \\
\hline Health-care system & $10(22 \%)$ \\
\hline No statement & $6(13 \%)$ \\
\hline \multicolumn{2}{|l|}{ Time horizon } \\
\hline Lifetime & $17(37 \%)$ \\
\hline$\geq 20$ years & $8(17 \%)$ \\
\hline $3-10$ years & $2(4.3 \%)$ \\
\hline$\leq 3$ years & $13(28 \%)$ \\
\hline Others $^{9}$ & $2(4 \%)$ \\
\hline No statement & $4(9 \%)$ \\
\hline \multicolumn{2}{|l|}{ Type of analysis } \\
\hline Cost-utility analysis & $43(93 \%)$ \\
\hline Cost-benefit analysis & $1(2 \%)$ \\
\hline Others $^{h}$ & $2(4 \%)$ \\
\hline \multicolumn{2}{|l|}{ Patient age range } \\
\hline$\geq 60$ years old & $22(48 \%)$ \\
\hline$<60$ years old & $5(11 \%)$ \\
\hline Not specified & $19(41 \%)$ \\
\hline
\end{tabular}

This table included all the studies while some categories did not add up to $100 \%$ due to rounding.

ACEls angiotensin-converting enzyme inhibitors.

aThe country of the study target population.

${ }^{b}$ Other countries/regions including Serbia, China, Croatia, Europe, Korea, New Zealand, Slovenia, Thailand, UK, UK and Sweden, United States and Canada. Each had one study reported.

'Other diseases or subgroups defined in the studies including arterial sclerosis cardiovascular disease, acute ischemic stroke, chronic heart disease, chronic heart failure, cardiovascular disease (general), coronary artery disease, stable coronary artery disease, hypertension, ST-elevated myocardial infarction, stroke, and acute myocardial infarction. Two studies limited analysis to those undergoing specific procedures (mechanical heart valve replacement and percutaneous coronary intervention $(\mathrm{PCI}))$.

${ }^{\mathrm{d}}$ Other drug categories: ivabradine and diuretics.

${ }^{e}$ Combinations of funding support including public and NPO, private and NPO, public and private.

${ }^{f}$ Payer perspective including both public payer and private payer

gStudy follow-up time was designed from a certain starting date to finishing date, and varied for each individual.

${ }^{h}$ Other types including descriptive summary and simulation estimation of costs. 


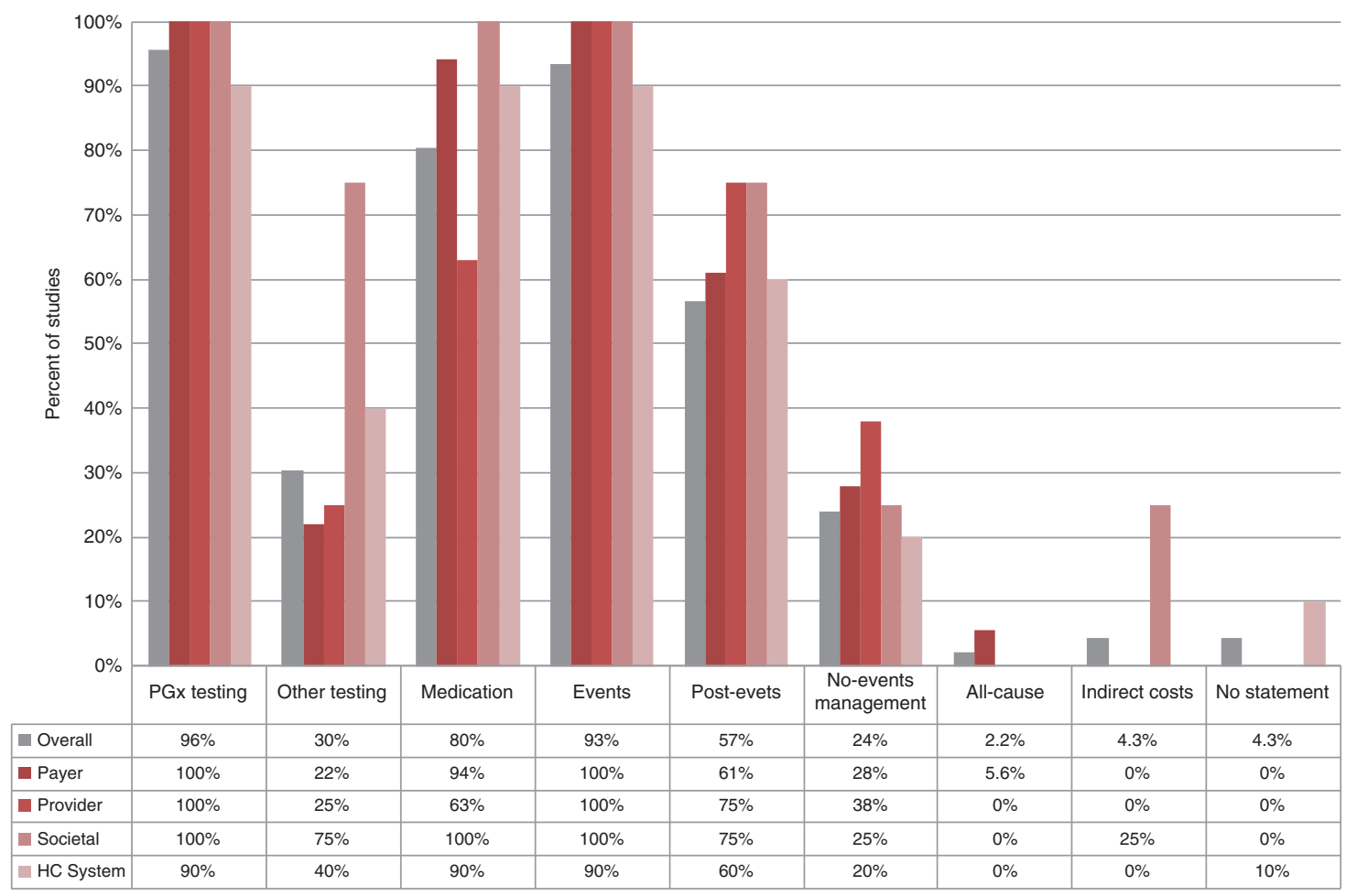

Fig. 2 Cost categories included in the study. Costs included in the studies were categorized into these major types: pharmacogenomics (PGx) testing, other types of testing (e.g., international normalized ratio [INR] monitoring, platelet function tests), medications (e.g., warfarin, clopidogrel), event-related costs (e.g., bleeding, stroke), postevent-related costs (long-term management costs), no-events costs (medical care costs for patients without adverse events), all-cause costs (any costs occurred after the treatment was initiated), and indirect costs (e.g., transportation, food). Several studies did not report the cost categories used in the calculation.

to patients undergoing certain surgical interventions (mechanical heart valve replacement and percutaneous coronary intervention). Six studies (13\%) were found to have no statement regarding any specific diagnosis or indication.

The two drug categories most studied were P2Y12 inhibitors (e.g., clopidogrel) and antithrombotic therapy (e.g., warfarin), accounting for $35 \%(n=16)$ and $43 \%(n=$ 20) of the studies respectively. A handful of studies investigated angiotensin-converting enzyme inhibitors (ACEIs) $(n=2,4.3 \%)$ and statins $(n=4,8.7 \%)$ (Table 1$)$. Only two studies (4.3\%) examined a panel of genetic testing, which was used to guide treatment for patients identified as having high risk. Virtually all studies $(n=44,96 \%)$ focused on PGx implementation after treatment plans were made or started (i.e., reactive genotyping), rather than a pre-emptive approach. Source of funding was reported for 31 of the 46 studies (67\%), which varied between public, private, nonprofit organizations (NPOs), and combinations thereof. Seven $(15 \%)$ studies reported that no funding support was used, while the remaining articles $(n=8,17 \%)$ did not report any information regarding funding resources.

\section{Summary of study strategies}

Most studies $(n=41,89 \%)$ used hypothetical cohorts, with model parameter estimates being derived from previously published randomized controlled trials and other literature.
The remaining studies were economic modeling studies using patient populations and model parameters from randomized controlled trials (RCTs) or observational studies. Eighteen (39\%) studies were conducted from a payer's or health insurance provider's perspective (private and public payers). The rest of the studies varied across the health-care system, health-care provider, and societal perspectives, with six (13\%) studies failing to specify the perspective used (Table 1). The time horizons used in the included cost-effectiveness studies were considerably diverse: about $37 \%(n=17)$ used lifetime models, $17 \%(n=8)$ used a 20-year or longer horizon, while $28 \%(n=13)$ used a 3-year or shorter time frame. Four $(8.7 \%)$ studies did not report a time horizon. Effectiveness was most commonly measured using quality-adjusted life-years (QALYs) ( $n=34,74 \%$ of studies).

The cost categories included in the economic model varied across studies. Most included the cost of PGx testing ( $n=44$, 96\%); one-time event costs, such as stroke or bleeding events ( $n=43,93 \%)$; and medication costs $(n=37,80 \%)$ (Fig. 2). Over half $(n=26,57 \%)$ of the studies included postevent or follow-up costs, and 30\% $(n=14)$ incorporated costs for other types of testing (e.g., platelet function testing). Several $(n=11,24 \%)$ studies used cost estimates for management of patients without events. Only two studies (4.3\%) incorporated indirect costs (e.g., transportation, food), and one (2.2\%) study used all-cause costs. 
All 46 studies used one-way deterministic sensitivity analyses to account for uncertainties in the model parameter estimates, and 33 studies additionally used probabilistic sensitivity analyses to explore different willingness-to-pay thresholds.

\section{Comparative-effectiveness results}

Thirty-one studies (67\%) found PGx testing to be a costeffective method in the treatment of CVD. Nine $(20 \%)$ conversely reported $\mathrm{PGx}$ testing as unlikely to be costeffective compared with standard care, and the remaining six (13\%) studies did not state any definitive conclusion. For those studies with no definitive conclusions, the author(s) reported conflicting results across treatment arms or from sensitivity analyses, indicating that cost-effectiveness was dependent upon parameter specification in the model. For studies that showed PGx testing to be cost-effective against the standard of care or referent therapy $(n=31), 81 \%(n=$ $25)$ found that PGx-guided approaches were the most costeffective option of all treatment arms examined. The other six studies focused on warfarin and clopidogrel and found alternative therapies that outperformed PGx-guided treatments (i.e., universal ticagrelor and dabigatran) (Supplemental Table S2).

We examined the cost-effectiveness conclusions by specific drug treatments and study perspectives (Table 2). PGx-guided treatment for the drugs clopidogrel and warfarin were most frequently studied, each accounting for 16 (35\%) of the 46 studies included in this review. PGx-guided clopidogrel treatment was cost-effective in the majority of these 16 studies $(n=13,81 \%)$ and across all perspectives. The evidence for PGx-guided warfarin treatment was not as strong, with almost half of the studies $(n=7,44 \%)$ concluding no costeffectiveness or mixed evidence, particularly from the societal perspective. All studies examining PGx-guided statin therapy $(n=4)$ supported its cost-effectiveness from the health-care system and payer perspectives; however, our review did not find any studies that assessed the cost-effectiveness of PGxguided statin therapy from the provider and societal perspectives. Results for the remaining treatments were limited and unclear. Only two studies examined panels of genetic testing: one of which found that PGx testing using a cardiovascular risk panel was not cost-effective; the other investigated a collection of eight gene-drug associations and was unable to make any definitive statement regarding the value of PGx testing.

We further summarized results for disease diagnosis or condition being studied most (ACS and AF, Supplemental Table S1). Of the 13 ACS studies, 10 (77\%) reported PGxguided therapy to be cost-effective. Seven (54\%) of these studies found PGx testing to be the dominant strategy (higher QALY while lower costs) when compared with no PGx testing. For the nondominant results, the incremental costeffectiveness ratios (ICERs) ranged widely from US\$196 to near US\$70,000 per QALY gained. Evidence among studies focusing on AF was only slightly less certain. Nine (64\%) of the 14 studies showed PGx to be cost-effective, with two identifying PGx testing as dominant. In the remaining studies, the ICERs ranged from less than US $\$ 3000$ to US $\$ 13,810$.

\section{Variation in results across study characteristics}

To investigate potential factors influencing the study conclusions, we summarized cost-effectiveness across model perspectives, funding mechanisms, and countries (Fig. 3a-c). The results showed variation across model perspectives. Studies conducted from the health-care provider, health-care payer, and health-care system perspectives had a high proportion of studies concluding PGx as a cost-effective strategy $(n=7 / 8$, $88 \% ; n=14 / 18,78 \%$; and $n=7 / 10,70 \%$ respectively), while only one study $(25.0 \%)$ from the societal perspective concluded cost-effectiveness. Cost-effectiveness was concluded in one-third $(n=2 / 6)$ of studies without a perspective identified. There was less variability across funding mechanism. Studies funded by public and private funding sources, as well as those with combinations of funding or no specific statement regarding funding, had similar proportions of costeffective studies $(n=12 / 17,71 \% ; n=4 / 5,80 \% ; n=4 / 7,57 \%$ and $n=5 / 8,63 \%$ respectively). Approximately $86 \%(n=6 / 7)$ of studies without funding support found PGx to be costeffective. Neither of the two studies funded by NPOs was able to find PGx testing to be a cost-effective approach. Evidence of cost-effectiveness of PGx testing was found across all countries of origin of the studies, although the strength of evidence varied. PGx testing was found to be cost-effective in approximately $62 \%(13 / 21)$ of US studies. Almost 20\% (4/21) of the US studies reported conflicting or mixed findings and could not definitively state a recommendation. Both studies $(n=2)$ from Australia were able to conclude that PGx is costeffective. Three-quarters of Canadian studies $(n=3$ of 4$)$ and half of studies from the Netherlands (3/6) also found supportive evidence for PGx-guided treatment. Evidence from the remaining other countries of origin had a high proportion of cost-effective studies ( $n=9$ of $10,90 \%)$.

\section{Quality of reporting}

Quality scores assessed using the QHES framework ranged from 53 to 100. Twenty-four studies (52\%) achieved a perfect quality reporting score of 100 , with an additional 14 (30\%) studies having scores between 91 and 97 . All 46 studies clearly stated their objective (QHES criteria 1) and primary outcomes of interest (QHES criteria 10). Subgroup analyses were not commonly performed in this group of literature, making QHES criteria 4 not applicable in most cases. Quality scores were not docked points in this situation. Other highly reported QHES elements included health outcomes (criteria 11), recommendations (criteria 15), and data abstraction (criteria 7). Variability was found in the level of reporting for a handful of QHES elements. Design elements critical in conducting economic evaluations (e.g., time horizon, discounting, parameter uncertainty, etc.) were underreported. Furthermore, nine studies (20\%) failed to report whether any source of funding was used to support the project and what sources of bias may be present in the study, as well as the 
Table 2 Evidence map of study conclusions regarding the cost-effectiveness of PGx-guided testing

\begin{tabular}{|c|c|c|c|c|c|c|c|}
\hline \multirow[b]{3}{*}{ Drug } & \multirow{3}{*}{$\begin{array}{c}\text { Number } \\
\text { of } \\
\text { Studies }\end{array}$} & \multicolumn{6}{|c|}{ Studies That Found PGx To Be Cost-Effective (\%)* } \\
\hline & & \multirow[b]{2}{*}{ Overall } & \multicolumn{5}{|c|}{ By Perspective } \\
\hline & & & $\begin{array}{c}\text { Healthcare } \\
\text { system }\end{array}$ & Payer & Provider & Societal & $\begin{array}{c}\text { No } \\
\text { Statement }\end{array}$ \\
\hline Clopidogrel ${ }^{23,42-55}$ & 16 & & $66.7 \%$ & & $-100 \%$ & $-100 \%$ & $-100 \%$ \\
\hline Warfarin ${ }^{53,56-70}$ & 16 & & $-75.0 \%$ & & $-66.7 \%$ & (-) $0 \%$ & (1-0\% \\
\hline Statin $^{71-74}$ & 4 & & $-100 \%$ & $-100 \%$ & & & \\
\hline $\begin{array}{l}\text { Coumarin } \\
\text { Derivatives }^{\dagger} \text { 75-77 }\end{array}$ & 3 & $56.7 \%$ & $-50.0 \%$ & & & & $-100 \%$ \\
\hline $\operatorname{ACEI}^{\ddagger 78,79}$ & 2 & (1)-0\% & & & & & (1) $\%$ \\
\hline Ivabradine $^{80}$ & 1 & $-100 \%$ & & $-100 \%$ & & & \\
\hline NOAC $\$ 81$ & 1 & $-100 \%$ & & $-100 \%$ & & & \\
\hline Diuretics $^{82}$ & 1 & $-100 \%$ & & $-100 \%$ & & & \\
\hline Drug panel ${ }^{83,84}$ & 2 & (-) $0 \%$ & & (1) $0 \%$ & & & (1) $0 \%$ \\
\hline
\end{tabular}

ACEl angiotensin-converting enzyme inhibitor, NOAC novel oral anticoagulant, $P G \times$ pharmacogenomics.

aThe size of the pie chart reflects the number of studies captured, and the shaded area reflects the proportion of studies that reported PGx-guided treatments to be cost-effective. Cells that are empty suggest that no evidence was found for the drug from the corresponding perspective.

${ }^{\mathrm{b}}$ Coumarin derivatives, including phenprocoumon and acenocoumarol.

Includes direct factor $\mathrm{Xa}$ inhibitors (apixaban, rivaroxaban, darexaban, edoxaban), direct thrombin inhibitors (dabigatran).

magnitude and direction of that bias. Additionally, seven studies (15\%) did not report the study perspective from which the economic evaluation was conducted, and cost inputs were often not described clearly. A description of each QHES criterion and the number of articles missing each element is reported in Supplemental Table S2.

\section{DISCUSSION}

Our study results suggest that the evidence on the costeffectiveness of implementing PGx testing in the treatment of CVD is mixed, though generally supportive for select gene-drug relationships (e.g., warfarin-CYP2C19/VKORC1 and clopidogrel-CYP2C19). Much of the available empirical evidence has been published in just the past decade, demonstrating the infancy of this field. The majority of studies targeted US patients with ACS or AF, demonstrating supportive evidence for ACS (clopidogrel-CYP2C19) and limited information for AF (warfarin-CYP2C19/VKORC1). Additionally, the economic value of genetic panel and preemptive testing is rarely examined. Cost categories included in the economic models varied across studies, which may have influenced the cost-effectiveness results reported. Sensitivity analyses conducted in these studies suggested significant uncertainties regarding the cost-effectiveness of PGx-based CVD care. Thus, our study shows that the cost-effectiveness of PGx-guided CVD care has not been unequivocally established.

The findings of our study align closely with previously published reviews in the PGx space. Berm et al. showed that most economic evaluations of PGx-guided treatment indicate favorable cost-effectiveness, with an average QHES score of 76; however, this study was not CVD focused. ${ }^{31}$ Verschuren 
a

Cost-Effectiveness of PGx testing by study perspectives

$\square$ Yes $\square$ No $\square$ No definite statement

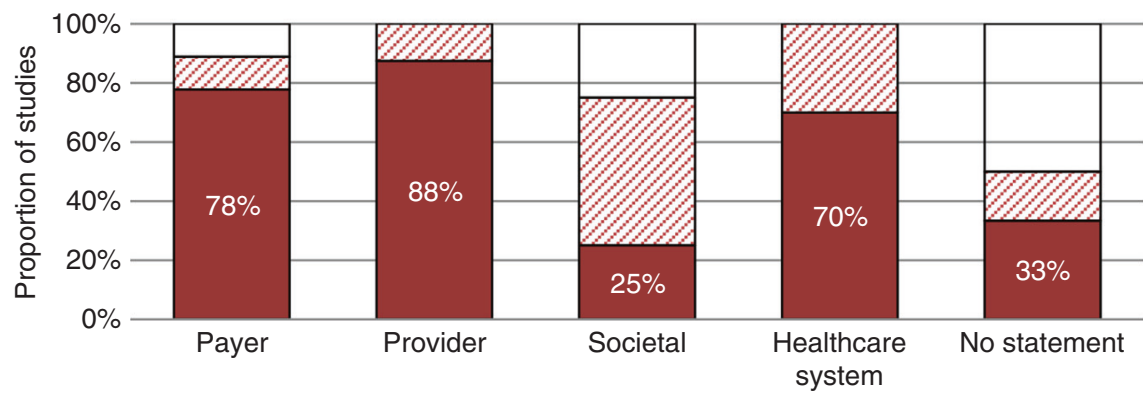

Study perspective

b

Cost-Effectiveness of PGx testing by funding support

$\square$ Yes $\square$ No $\square$ No definite statement

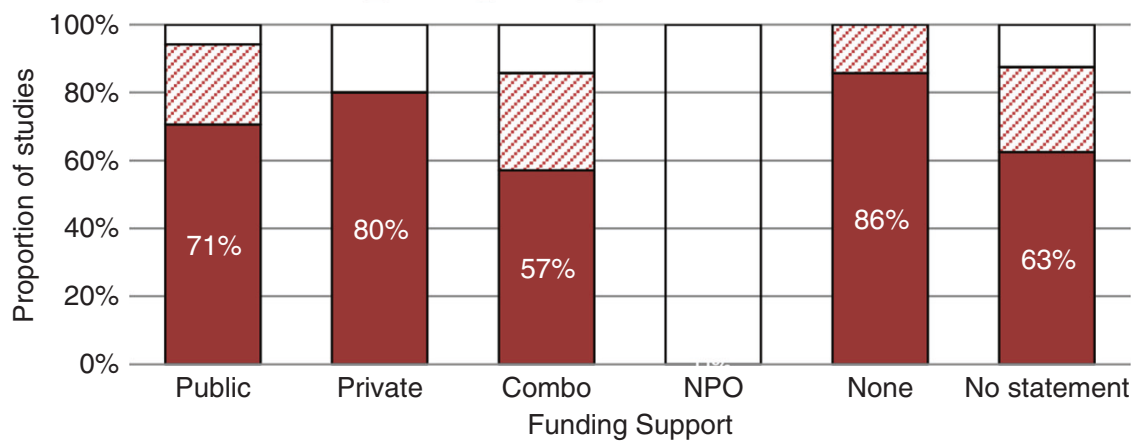

C Cost-Effectiveness of PGx testing by study countries

$\square$ Yes $\square$ No $\square$ No definite statement

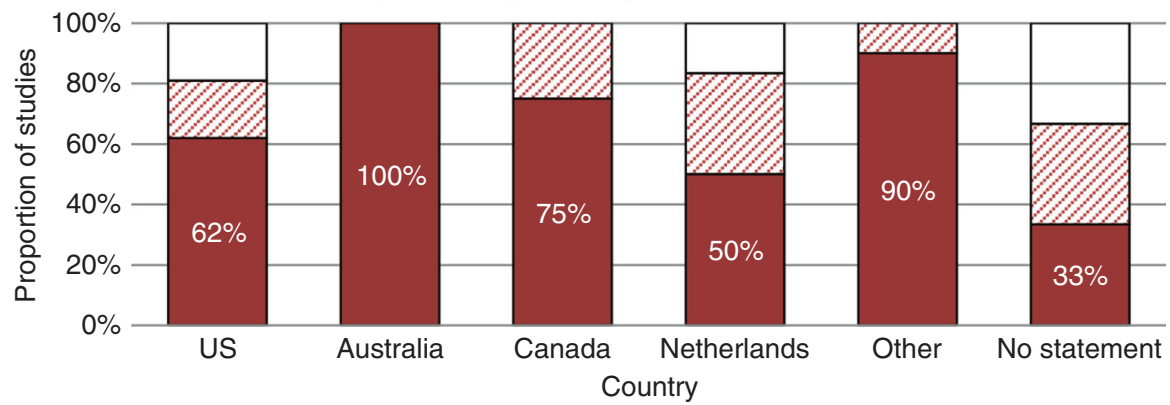

Fig. 3 Comparison of cost-effectiveness with selected study characteristics. a Perspectives held in the studies, including payer (both public and private), provider, societal, and health-care system. Studies that did not report the perspectives used were categorized as no statement. $\mathbf{b}$ Funding support types. Combination types included private + public, public + nonprofit organization (NPO), and private + NPO. c Country/regions of the study population. Other countries/regions included Serbia, China, Croatia, Europe, Korea, New Zealand, Slovenia, Thailand, UK, UK and Sweden, United States and Canada. PGx pharmacogenomics.

et al. demonstrated varying strength of evidence across different CVD drug classes and, similarly, found the strongest evidence among antiplatelet and anticoagulant therapies. ${ }^{32}$ However, this study did not specifically examine economic value, but rather focused on associations between gene variants and risk of adverse drug events or ineffectiveness.

Other studies also reported the lack of a standard approach in study design across economic evaluations for PGx testing, including frequent underreporting of important components (e.g., bias, funding sources, etc.), high variability in cost inputs, substantial variation in study strategies across the existing evidence, and limited representation of the societal perspective, which is an approach recommended by the second panel on cost-effectiveness in health and medicine. ${ }^{29,33-35} \mathrm{We}$ also found that cost categories used across study perspectives were diverse, which may have contributed to the varying study conclusions. As illustrated in Fig. 2, costs for PGx testing, as well as costs for disease-related adverse outcome events, were included in almost all studies across perspectives. However, fewer than $30 \%$ of the studies from the provider's and payer's perspectives adopted costs for other types of medical exams. Medication costs were well 
represented from the payer, societal, and health-care system perspectives, but not as consistently included among studies conducted from the provider's perspective. A small number of studies included costs for chronic disease management with no events, which is a very significant part of health-care costs. As expected, no studies conducted from the payer, provider, or health-care system perspectives accounted for indirect costs (e.g., transportation, food). However, unlike we expected, fewer than one-third of the studies using societal perspective included indirect costs, potentially resulting in underestimation of cost impacts in those studies. Thus, future studies should account for these data gaps as well as adhere to standard approaches for conducting economic evaluations, which will strengthen the evidence base on cost-effectiveness of PGx-guided therapy in CVD, and also foster meaningful comparisons between different studies. Furthermore, as PGxguided drugs become more widely used in practice, studies based on real-world databases such as large health-care claims or registry databases should provide long-term outcomes, both on cost and effectiveness measures, which will help provide robust evidence on long-term cost-effectiveness of PGx-guided CVD therapy.

We have shown that notable gaps persist in the evaluation of PGx-guided treatment. Panel testing (i.e., testing for multiple actionable gene-drug relationships) is gaining more attention in research, and is being adopted into clinical practice, as very few patients carry only a single genetic variation. Thus, panel testing is an important method for identifying risk factors and predicting drug responses. ${ }^{12,36,37}$ This move toward panel testing also implies a need for preemptive genetic testing (i.e., having the genetic information available at the point of care versus reactively genotyping). This is an important area to consider because pre-emptive testing results have been shown to be highly utilized for prescribing and associated with $\mathrm{PGx}$-indicated medication changes. ${ }^{38}$ Furthermore, pre-emptive testing has potential to improve patient satisfaction and generate significant reductions in the utilization of reactive genetic testing. ${ }^{39,40}$ Despite the benefits of pre-emptive and panel testing, reactive genotyping strategies are dominant in clinical practice and the economic evaluations of panel and pre-emptive PGx testing are underrepresented in the empirical literature. This may be due to the risk that, for many individuals, genetic variations may have limited clinical impact due to the possibility of never developing the disease. In which case, the health-care system will likely bear the costs of testing, the patient may become subject to potential harm from treatment in the case of false-positive results, and additional efforts and costs associated with PGx information storage may be unfruitful. For these reasons, pre-emptive testing may still be a less attractive approach. However, further studies are needed to fill in significant gaps in evidence that can help us understand the existing barriers, including lack of guidance on how to use PGx information and skepticism from payers regarding the effect of $\mathrm{PGx}$-guided therapy on outcomes, aversion to adverse drug events, and evidence of long-term benefits. ${ }^{41,42}$ Therefore, the value of pre-emptive panel testing should be investigated and the barriers to implementing these tests should be addressed.

We have shown that the majority of empirical evidence continues to focus on only two gene-drug relationships, CYP2C9/VKORC1 (warfarin) and CYP2C19 (clopidogrel). Studies dominantly supported the cost-effectiveness of CYP2C19 (clopidogrel), especially from the provider and societal perspectives, while studies conducted from the payer and health-care system perspectives were less supportive. The evidence for CYP2C9/VKORC1 (warfarin) was more diverse with less support for cost-effectiveness from the societal and provider perspectives. Although the number of studies examined was small, our study provides supportive evidence that implementing PGx-guided treatment in ACS patients being prescribed clopidogrel might be beneficial from multiple perspectives. On the other hand, implementing PGx-guided treatment approaches for AF patients receiving warfarin needs more careful evaluation from different perspectives. However, as stated earlier, the diversity in our results could be due to multiple factors, including the costs of alternative medicine (e.g., ticagrelor versus clopidogrel, novel oral anticoagulant [NOAC] versus warfarin), the study perspectives used, the payment mechanism of the healthcare system, and long-term costs of disease management.

Besides the drug-gene pairs studied in the current literature, the FDA has outlined numerous other CVD treatments with PGx interactions that have emerged in the market. ${ }^{43}$ Future research has the opportunity to build upon this literature by examining new drug-gene associations for cost-effectiveness.

This review has the strength of examining the costeffectiveness of PGx-guided treatment for CVDs from a broad scope compared with focusing on one specific disease or gene. Consequently, this study enabled the synthesis of PGx-related findings for an entire class of diseases, making this study more widely informative and relevant. This review included all studies targeting patients internationally, which allowed us to have a broader view to examine potential differences due to geography and national policy. ${ }^{44-47} \mathrm{We}$ focused on PGx testing with an actionable gene-drug relationship used to guide treatment decisions, making this review of evidence useful for clinical practice and medical decision making.

This review is constrained by the quality of the studies included in the sample, which may lead to inaccurate inferences and synthesis, particularly considering the substantial effect these parameters have on the study conclusions. The quality of reporting was high for most studies, but some studies did fail to clearly define and report on the perspectives, cost inputs, or study design elements of the economic model. Additionally, a handful of studies in our sample used models built from observational data, which may have resulted in the use of biased parameter estimates. Another potential limitation is the fact that approximately $89 \%$ of the studies on which our review was based used 
hypothetical cohorts. While the use of hypothetical cohort is a standard practice in decision analytic modeling for costeffectiveness analyses, interpretation of the study results needs to account for this preponderance of hypothetical cohorts, as results may differ when replicated in real cohorts. Furthermore, this study is also limited by summarizing results from published studies only, thus potentially missing important unpublished data.

In conclusion, PGx-guided drug prescribing holds enormous potential for individualizing CVD management and reducing the chances of drug-related adverse events. This review finds overwhelming evidence of cost-effectiveness among the most commonly studied treatments (e.g., clopidogrel-CYP2C19, warfarin--CYP2C9/VKORC1), while evidence is more limited in others (e.g., ACE inhibitors, NOACs, CVD panels). Extant studies were mainly focused on traditional treatments while new gene-drug studies are scarce, and therefore need to be evaluated in the future. Assessing costeffectiveness of genetic panel testing and pre-emptive PGx testing will be crucial for health systems contemplating implementation of these approaches. This review identifies important strengths, limitations, and challenges that should be considered before clinical adoption of this strategy, and can be used to inform health-care systems aiming to implement PGx to improve CVD care.

\section{SUPPLEMENTARY INFORMATION}

The online version of this article (https://doi.org/10.1038/s41436019-0667-y) contains supplementary material, which is available to authorized users.

\section{ACKNOWLEDGEMENTS}

The value of pharmacogenomics has not been unequivocally established, particularly surrounding pre-emptive and panel testing approaches. Standardized modeling approaches and high-quality reporting are needed. This research was partially supported by a research grant from Mayo Clinic Robert D. and Patricia E. Kern Center for the Science of Health Care Delivery and Mayo Clinic Center for Individualized Medicine. The authors affirm that this paper is an honest, accurate, and transparent account of the study being reported; that no important aspects of the study have been omitted; and that any discrepancies from the study as planned have been explained. The views expressed in the article are those of the authors and do not necessarily reflect the views of Mayo Clinic.

\section{DISCLOSURE}

None of the authors have any potential conflicts of interest with respect to the authorship and/or publication of this article. None of the authors reported any financial relationships with any organizations that might have an interest in the submitted work in the previous three years. RW and LW are co-founders of and stock-holders in OneOme LLC, a pharmacogenomic decision support company. There are no other relationships or activities that could appear to have influenced the submitted work.
Publisher's note Springer Nature remains neutral with regard to jurisdictional claims in published maps and institutional affiliations.

\section{REFERENCES}

1. Weinshilboum R. Inheritance and drug response. $N$ Engl J Med. 2003;348:529-537.

2. Weinshilboum R, Wang L. Pharmacogenomics: bench to bedside. Nat Rev Drug Discov. 2004;3:739.

3. Payne K, Gavan SP, Wright SJ, Thompson AJ. Cost-effectiveness analyses of genetic and genomic diagnostic tests. Nat Rev Genet. 2018;19:235.

4. World Health Organization. Cardiovacular diseases (CVDs). 2017. https:// www.who.int/news-room/fact-sheets/detail/cardiovascular-diseases-(cvds).

5. American Heart Assocation. Cardiovascular disease: a costly burden for America, projections through 2035. CVD Burden Report. 2017. https:// healthmetrics.heart.org/wp-content/uploads/2017/10/CardiovascularDisease-A-Costly-Burden.pdf. Accessed February 29, 2018.

6. Johnson JA, Cavallari LH. Pharmacogenetics and cardiovascular diseaseimplications for personalized medicine. Pharmacol Rev. 2013;65:987-1009.

7. Peters BJM, Klungel OH, de Boer A, Ch Stricker BH, Maitland-van der Zee A-H. Pharmacogenetics of cardiovascular drug therapy. Clin Cases Miner Bone Metab. 2009;6:55-65.

8. Evans WE, McLeod HL. Pharmacogenomics-drug disposition, drug targets, and side effects. N Engl J Med. 2003;348:538-549.

9. Lee M-S, Flammer AJ, Lerman LO, Lerman A. Personalized medicine in cardiovascular diseases. Korean Circ J. 2012;42:583-591.

10. Joyner MJ. Precision medicine, cardiovascular disease and hunting elephants. Prog Cardiovasc Dis. 2016;58:651-660.

11. King KR, Grazette LP, Paltoo DN, et al. Point-of-care technologies for precision cardiovascular care and clinical research: National Heart, Lung, and Blood Institute Working Group. JACC Basic Transl Sci. 2016;1:73-86.

12. Ji Y, Skierka JM, Blommel JH, et al. Preemptive pharmacogenomic testing for precision medicine: a comprehensive analysis of five actionable pharmacogenomic genes using next-generation DNA sequencing and a customized CYP2D6 genotyping cascade. J Mol Diagn. 2016;18:438-445.

13. Carpenter JS, Rosenman MB, Knisely MR, Decker BS, Levy KD, Flockhart DA. Pharmacogenomically actionable medications in a safety net health care system. SAGE Open Med. 2016;4:2050312115624333.

14. Schuck RN, Grillo JA. Pharmacogenomic biomarkers: an FDA perspective on utilization in biological product labeling. AAPS J. 2016;18:573-577.

15. Trompet S, Postmus I, Slagboom P, et al. Non-response to (statin) therapy: the importance of distinguishing non-responders from non-adherers in pharmacogenetic studies. Eur J Clin Pharmacol. 2016;72:431-437.

16. US Food and Drug Administration. Table of pharmacogenomic biomarkers in drug labeling. 2019. https://www.fda.gov/drugs/scienceresearch-drugs/table-pharmacogenomic-biomarkers-drug-labeling. Accessed August 29, 2019.

17. Mega JL, Hochholzer W, Frelinger AL, et al. Dosing clopidogrel based on CYP2C19 genotype and the effect on platelet reactivity in patients with stable cardiovascular disease. JAMA. 2011;306:2221-2228.

18. Mega JL, Simon T, Collet J-P, et al. Reduced-function CYP2C19 genotype and risk of adverse clinical outcomes among patients treated with clopidogrel predominantly for PCl: a meta-analysis. JAMA. 2010;304:1821-1830.

19. Stergiopoulos K, Brown DL. Genotype-guided vs clinical dosing of warfarin and its analogues: meta-analysis of randomized clinical trials. JAMA Intern Med. 2014;174:1330-1338.

20. Kimmel SE, French B, Kasner SE, et al. A pharmacogenetic versus a clinical algorithm for warfarin dosing. N Engl J Med. 2013;369:2283-2293.

21. Holmes MV, Perel P, Shah T, Hingorani AD, Casas JP. CYP2C19 genotype, clopidogrel metabolism, platelet function, and cardiovascular events: a systematic review and meta-analysis. JAMA. 2011;306:2704-2714.

22. Bauer T, Bouman HJ, van Werkum JW, Ford NF, Ten Berg JM, Taubert D. Impact of CYP2C 19 variant genotypes on clinical efficacy of antiplatelet treatment with clopidogrel: systematic review and meta-analysis. BMJ. 2011;343:d4588

23. Pirmohamed $M$, Burnside $G$, Eriksson $N$, et al. A randomized trial of genotype-guided dosing of warfarin. N Engl J Med. 2013;369:2294-2303.

24. Kazi DS, Garber AM, Shah RU, et al. Cost-effectiveness of genotypeguided and dual antiplatelet therapies in acute coronary syndrome. Ann Intern Med. 2014;160:221-232.

25. Musunuru K. Personalized genomes and cardiovascular disease. Cold Spring Harb Perspect Med. 2015;5:a014068. 
26. Ioannidis JP. Prediction of cardiovascular disease outcomes and established cardiovascular risk factors by genome-wide association markers. Circ Cardiovasc Genet. 2009;2:7-15.

27. Billings J, Racsa PN, Bordenave K, Long CL, Ellis JJ. The impact of realworld cardiovascular-related pharmacogenetic testing in an insured population. Int J Clin Pract. 2018;72:e13088.

28. van der Laan SW, Harshfield EL, Hemerich D, Stacey D, Wood AM, Asselbergs FW. From lipid locus to drug target through human genomics. Cardiovasc Res. 2018;114:1258-1270.

29. Sanders GD, Neumann PJ, Basu A, et al. Recommendations for conduct, methodological practices, and reporting of cost-effectiveness analyses: second panel on cost-effectiveness in health and medicine. JAMA. 2016;316:1093-1103

30. Chiou C-F, Hay JW, Wallace JF, et al. Development and validation of a grading system for the quality of cost-effectiveness studies. Med Care. 2003;41:32-44.

31. Berm EJ, Looff $M$, Wilffert $B$, et al. Economic evaluations of pharmacogenetic and pharmacogenomic screening tests: a systematic review. Second update of the iterature. PLoS ONE. 2016;11:e0146262.

32. Verschuren JJ, Trompet S, Wessels JA, et al. A systematic review on pharmacogenetics in cardiovascular disease: is it ready for clinical application? Eur Heart J. 2012;33:165-175.

33. Wong WB, Carlson JJ, Thariani R, Veenstra DL. Cost effectiveness of pharmacogenomics: a critical and systematic review. Pharmacoeconomics. 2010;28:1001-1013.

34. Beaulieu M, de Denus S, Lachaine J. Systematic review of pharmacoeconomic studies of pharmacogenomic tests. Pharmacogenomics. 2010;11:1573-1590.

35. Djalalov S, Musa Z, Mendelson M, Siminovitch K, Hoch J. A review of economic evaluations of genetic testing services and interventions (2004-9). Genet Med. 2011;13:89-94.

36. Ehret GB, Ferreira T, Chasman DI, et al. The genetics of blood pressure regulation and its target organs from association studies in 342,415 individuals. Nat Genet. 2016:48:1171

37. Weitzel KW, Cavallari LH, Lesko $\amalg$. Preemptive panel-based pharmacogenetic testing: the time is now. Pharm Res. 2017;34:1551-1555.

38. O'Donnell PH, Wadhwa N, Danahey K, et al. Pharmacogenomics-based point-of-care clinical decision support significantly alters drug prescribing. Clin Pharmacol Ther. 2017;102:859-869.

39. McKillip RP, Borden BA, Galecki P, et al. Patient perceptions of care as influenced by a large institutional pharmacogenomic implementation program. Clin Pharmacol Ther. 2017;102:106-114.

40. Van Driest SL, Shi Y, Bowton EA, et al. Clinically actionable genotypes among 10,000 patients with preemptive pharmacogenomic testing. Clin Pharmacol Ther. 2014;95:423-431.

41. Dunnenberger HM, Crews KR, Hoffman JM, et al. Preemptive clinical pharmacogenetics implementation: current programs in five US medical centers. Annu Rev Pharmacol Toxicol. 2015;55:89-106.

42. Keeling NJ, Rosenthal MM, West-Strum D, Patel AS, Haidar CE, Hoffman JM. Preemptive pharmacogenetic testing: exploring the knowledge and perspectives of US payers. Genet Med. 2019;21:1224-1232.

43. Larsen TB, Skjøth F, Nielsen PB, Kjældgaard JN, Lip GY. Comparative effectiveness and safety of non-vitamin $\mathrm{K}$ antagonist oral anticoagulants and warfarin in patients with atrial fibrillation: propensity weighted nationwide cohort study. BMJ. 2016;353:i3189.

44. Relling MV, Evans WE. Pharmacogenomics in the clinic. Nature. 2015:526:343.

45. Wang Y, Yan BP, Liew D, Lee VWY. Cost-effectiveness of cytochrome P450 2C19*2 genotype-guided selection of clopidogrel or ticagrelor in Chinese patients with acute coronary syndrome. Pharmacogenomics J. 2018;18:113-120.

46. Okere AN, Ezendu K, Berthe A, Diaby V. An evaluation of the costeffectiveness of comprehensive MTM integrated with point-of-care phenotypic and genetic testing for U.S. elderly patients after percutaneous coronary intervention. J Manag Care Spec Pharm. 2018;24:142-152.

47. Jiang M, You JHS. CYP2C 19 LOF and GOF-guided antiplatelet therapy in patients with cute coronary syndrome: a cost-effectiveness analysis. Cardiovasc Drugs Ther. 2017;31:39-49.

48. Jiang M, You JH. Cost-effectiveness analysis of personalized antiplatelet therapy in patients with acute coronary syndrome. Pharmacogenomics. 2016:17:701-713.

49. Johnson SG, Gruntowicz D, Chua T, Morlock RJ. Financial analysis of CYP2C19 genotyping in patients receiving dual antiplatelet therapy following acute coronary syndrome and percutaneous coronary intervention. J Manag Care Spec Pharm. 2015;21:552-557.

50. Patel V, Lin F-J, Ojo O, et al. Cost-utility analysis of genotype-guided antiplatelet therapy in patients with moderate-to-high risk acute coronary syndrome and planned percutaneous coronary intervention. Pharm Pract (Granada). 2014;12:438.

51. Sorich MJ, Horowitz JD, Sorich W, Wiese MD, Pekarsky B, Karnon JD. Cost-effectiveness of using CYP2C19 genotype to guide selection of clopidogrel or ticagrelor in Australia. Pharmacogenomics. 2013;14:2013-2021.

52. Lala A, Berger JS, Sharma G, Hochman JS, Scott Braithwaite R, Ladapo JA. Genetic testing in patients with acute coronary syndrome undergoing percutaneous coronary intervention: a cost-effectiveness analysis. J Thromb Haemost. 2013;11:81-91.

53. Panattoni L, Brown PM, Te Ao B, Webster M, Gladding P. The cost effectiveness of genetic testing for CYP2C19 variants to guide thienopyridine treatment in patients with acute coronary syndromes: a New Zealand evaluation. Pharmacoeconomics. 2012;30:1067-1084.

54. Crespin DJ, Federspiel JJ, Biddle AK, Jonas DE, Rossi JS. Ticagrelor versus genotype-driven antiplatelet therapy for secondary prevention after acute coronary syndrome: a cost-effectiveness analysis. Value Health. 2011;14:483-491

55. Borse MS, Dong OM, Polasek MJ, Farley JF, Stouffer GA, Lee CR. CYP2C19-guided antiplatelet therapy: a cost-effectiveness analysis of 30day and 1-year outcomes following percutaneous coronary intervention. Pharmacogenomics. 2017;18:1155-1166.

56. Mitropoulou C, Fragoulakis V, Rakicevic LB, et al. Economic analysis of pharmacogenomic-guided clopidogrel treatment in Serbian patients with myocardial infarction undergoing primary percutaneous coronary intervention. Pharmacogenomics. 2016;21:21.

57. Deiman BALM, Tonino PAL, Kouhestani $K$, et al. Reduced number of cardiovascular events and increased cost-effectiveness by genotypeguided antiplatelet therapy in patients undergoing percutaneous coronary interventions in the Netherlands. Neth Heart J. 2016; 24:589-599.

58. Reese ES, Daniel Mullins C, Beitelshees AL, Onukwugha E. Costeffectiveness of cytochrome P450 2C19 genotype screening for selection of antiplatelet therapy with clopidogrel or prasugrel [erratum appears in Pharmacotherapy. 2012;32:581]. Pharmacotherapy. 2012;32:323-332

59. Verhoef TI, Redekop WK, Langenskiold S, et al. Cost-effectiveness of pharmacogenetic-guided dosing of warfarin in the United Kingdom and Sweden. Pharmacogenomics J. 2016;16:478-484.

60. Janzic A, Kos M. Cost effectiveness of novel oral anticoagulants for stroke prevention in atrial fibrillation depending on the quality of warfarin anticoagulation control. Pharmacoeconomics. 2015;33:395-408.

61. You JHS. Pharmacogenetic-guided selection of warfarin versus novel oral anticoagulants for stroke prevention in patients with atrial fibrillation: a cost-effectiveness analysis. Pharmacogenet Genomics. 2014;24:6-14.

62. Pink J, Pirmohamed $M$, Lane $S$, Hughes DA. Cost-effectiveness of pharmacogenetics-guided warfarin therapy vs. alternative anticoagulation in atrial fibrillation. Clin Pharmacol Ther. 2014;95:199-207.

63. Nshimyumukiza L, Duplantie J, Gagnon M, et al. Dabigatran versus warfarin under standard or pharmacogenetic-guided management for the prevention of stroke and systemic thromboembolism in patients with atrial fibrillation: a cost/utility analysis using an analytic decision model. Thromb J. 2013;11:14.

64. You JHS, Tsui KKN, Wong RSM, Cheng G. Cost-effectiveness of dabigatran versus genotype-guided management of warfarin therapy for stroke prevention in patients with atrial fibrillation. PLoS ONE. 2012;7: e39640.

65. Meckley LM, Gudgeon JM, Anderson JL, Williams MS, Veenstra DL. A policy model to evaluate the benefits, risks and costs of warfarin pharmacogenomic testing. Pharmacoeconomics. 2010;28:61-74.

66. Patrick AR, Avorn J, Choudhry NK. Cost-effectiveness of genotypeguided warfarin dosing for patients with atrial fibrillation. Circ Cardiovasc Qual Outcomes. 2009;2:429-436.

67. Eckman MH, Rosand J, Greenberg SM, Gage BF. Cost-effectiveness of using pharmacogenetic information in warfarin dosing for patients with nonvalvular atrial fibrillation. Ann Intern Med. 2009;150:73-83.

68. Kim D-J, Kim H-S, Oh M, Kim E-Y, Shin J-G. Cost effectiveness of genotype-guided warfarin dosing in patients with mechanical heart valve replacement under the fee-for-service system. Appl Health Econ Health Policy. 2017;15:657-667. 
69. Chong HY, Saokaew S, Dumrongprat K, et al. Cost-effectiveness analysis of pharmacogenetic-guided warfarin dosing in Thailand. Thromb Res. 2014;134:1278-1284.

70. You JHS, Tsui KKN, Wong RSM, Cheng G. Potential clinical and economic outcomes of CYP2C9 and VKORC1 genotype-guided dosing in patients starting warfarin therapy. Clin Pharmacol Ther. 2009;86:540-547.

71. Leey JA, McCabe S, Koch JA, Miles TP. Cost-effectiveness of genotypeguided warfarin therapy for anticoagulation in elderly patients with atrial fibrillation. Am J Geriatr Pharmacother. 2009;7:197-203.

72. McWilliam A, Lutter R, Nardinelli C. Healthcare impact of personalized medicine using genetic testing: an exploratory analysis for warfarin. Per Med. 2008;5:279-284.

73. You JHS, Chan FWH, Wong RSM, Cheng G. The potential clinical and economic outcomes of pharmacogenetics-oriented management of warfarin therapy-a decision analysis. Thromb Haemost. 2004; 92:590-597.

74. Parthan A, Leahy KJ, O'Sullivan AK, et al. Cost effectiveness of targeted high-dose atorvastatin therapy following genotype testing in patients with acute coronary syndrome. Pharmacoeconomics. 2013;31:519-531.

75. Mitchell D, Guertin JR, Dubois A, et al. A discrete event simulation model to assess the economic value of a hypothetical pharmacogenomics test for statin-induced myopathy in patients initiating a statin in secondary cardiovascular prevention. Mol Diagn Ther. 2018;22:241-254.

76. Mitchell D, Guertin JR, Iliza AC, Fanton-Aita F, LeLorier J. Economic evaluation of a pharmacogenomics test for statin-induced myopathy in cardiovascular high-risk patients initiating a statin. Mol Diagn Ther. 2017;21:95-105.

77. Kemp LK, Doran CM, Vos T, Hall W. Cost-effectiveness analysis of genetic screening for the Taq1B polymorphism in the secondary prevention of coronary heart disease. Expert Rev Pharmacoecon Outcomes Res. 2007;7:119-128.

78. Verhoef TI, Redekop WK, de Boer A, Maitland-van der Zee AH, EUPACT Group. Economic evaluation of a pharmacogenetic dosing algorithm for coumarin anticoagulants in The Netherlands. Pharmacogenomics. 2015;16:101-114.

79. Verhoef TI, Redekop WK, Veenstra DL, et al. Cost-effectiveness of pharmacogenetic-guided dosing of phenprocoumon in atrial fibrillation. Pharmacogenomics. 2013;14:869-883.

80. Schalekamp T, Boink GJJ, Visser LE, Stricker BHC, de Boer A, Klungel OH. CYP2C9 genotyping in acenocoumarol treatment: is it a cost-effective addition to international normalized ratio monitoring? Clin Pharmacol Ther. 2006;79:511-520.
81. Geenen JW, Baranova EV, Asselbergs FW, et al. Early health technology assessments in pharmacogenomics: a case example in cardiovascular drugs. Pharmacogenomics. 2017;18:1143-1153.

82. Oemrawsingh RM, Akkerhuis KM, Van Vark LC, et al. Individualized angiotensin-converting enzyme (ACE)-inhibitor therapy in stable coronary artery disease based on clinical and pharmacogenetic determinants: the PERindopril GENEtic (PERGENE) risk model. J Am Heart Assoc. 2016;5: e002688.

83. Iliza AC, Matteau A, Guertin JR, et al. A model to assess the costeffectiveness of pharmacogenomics tests in chronic heart failure: the case of ivabradine. Pharmacogenomics. 2016;10:10.

84. You JHS. Universal versus genotype-guided use of direct oral anticoagulants in atrial fibrillation patients: a decision analysis. Pharmacogenomics. 2015;16:1089-1100.

85. Meckley LM, Veenstra DL. Screening for the alpha-adducin Gly460Trp variant in hypertensive patients: a cost-effectiveness analysis. Pharmacogenet Genomics. 2006;16:139-147.

86. Billings J, Racsa PN, Bordenave K, Long CL, Ellis JJ. The impact of realworld cardiovascular-related pharmacogenetic testing in an insured population. Int J Clin Pract. 2018;72:e13088.

87. Arnaout R, Buck TP, Roulette P, Sukhatme VP. Predicting the cost and pace of pharmacogenomic advances: an evidence-based study. Clin Chem. 2013;59:649-657. Open Access This article is licensed under a Creative Commons
c) License, which permits any non-commercial use, sharing, adaptation, distribution and reproduction in any medium or format, as long as you give appropriate credit to the original author(s) and the source, provide a link to the Creative Commons license, and indicate if changes were made. If you remix, transform, or build upon this article or a part thereof, you must distribute your contributions under the same license as the original. The images or other third party material in this article are included in the article's Creative Commons license, unless indicated otherwise in a credit line to the material. If material is not included in the article's Creative Commons license and your intended use is not permitted by statutory regulation or exceeds the permitted use, you will need to obtain permission directly from the copyright holder. To view a copy of this license, visit http://creativecommons.org/licenses/by-nc-sa/4.0/.

(C) The Author(s) 2019 